\title{
"Commentary: The ageing as a therapeutic target for Alzheimer's disease"
}

\author{
José M. Brito-Armas and Rafael Castro-Fuentes* \\ Department of Basic Medical Sciences, University of La Laguna, Tenerife, Spain
}

Article Info

\section{Article Notes}

Received: July 12, 2017

Accepted: August 21, 2017

\section{${ }^{*}$ Correspondence:}

Dr. Rafael Castro-Fuentes,

Department of Basic Medical Sciences,

School of Health Sciences, Section Medicine,

University of La Laguna, 38200 Tenerife, Spain,

Tel.: +34-922 319360, Fax: +34-922 319397,

Email: jrcastro@ull.edu.es

(c) 2017 Castro-Fuentes R. This article is distributed under the terms of the Creative Commons Attribution 4.0 International License

\section{Keywords}

Aging

Anti-aging

Alzheimer's disease

Therapies
Alzheimer's disease (AD) is the most common form of dementia in the elderly and, in the absence of effective prevention and treatment, is estimated to affect 160 million people worldwide by $2050^{1}$, leading to potential bankruptcy of the Medicare system. Despite researchers great efforts to stall, slow, or even beat this devastating neurodegenerative condition, there are still no effective drugs available to the estimated 5.4 million Americans with the disease. Clinical trials in patients with $\mathrm{AD}$ have led to only modest positive outcomes or potentially serious adverse events in response to both active (Aß peptide with a conjugate to stimulate an immune response) or passive (administration of anti$A ß$ antibodies) administration ${ }^{2}$. Adverse effects associated with anti-Aß immuno- therapy include edema and microhemorrhages in the case of passive vaccines ${ }^{3,4}$ and aseptic meningoencephalitis with active vaccine approaches ${ }^{5}$. Despite these, both are still being explored in a number of clinical trials ${ }^{2}$.These accumulating failures call into question the promise of targeting the formation and occurrence of amyloid plaques as a viable approach for treating AD. The vast majority of research on $\mathrm{AD}$ continues to deal with a pathogenetic sequence that addresses the genesis and neuropathological consequences of abnormal amounts and types of beta amyloid peptides and associated abnormal forms of tau, gliosis and inflammatory responses.

So how do you break the chain? Are there other approaches that could give us valuable information before investing in human studies?

Qualitative neurocognitive differences between AD and normal aging clearly suggest that $\mathrm{AD}$ cannot be simply regarded as an accelerated aging process ${ }^{6}$. A more heuristic hypothesis, however, would embrace the hypothesis that there may be several distinct upstream pathogenetic pathways that are driven by one or more basic mechanisms of biological aging, all of which lead to a common downstream pathway of beta amyloidosis, tauopathies and inflammation?

Although average life expectancy has increased dramatically in the last 100 years, this has not been accompanied by an equivalent increase in healthy life expectancy, which has been termed healthspan ${ }^{8}$. Aging is the greatest threat to human health in the developed world. Research on the basic biology of aging aims to understand mechanisms that cause organisms to decline in function over time and lead to increasing risk of morbidity and mortality. Understanding why this relationship between age and disease exists and, ultimately, intervening in the aging process at a molecular level to promote healthy longevity is a primary goal of anti-aging research ${ }^{9}$.This is, of course, intimately connected to pathology because aging promotes disease. Most leading causes of mortality in 
developed nations share a single greatest risk factor, and it isn't how much you eat, drink, smoke, or exercise; it's how old you are ${ }^{10}$. Alzheimer' disease, along with many others chronic diseases, all show an exponential increase in risk with age over much of the human life $\operatorname{span}^{10,11,12}$. Since most of these chronic diseases appear when the body ages, does not it make sense to assume that they share common elements that should be combated? And is not that tantamount to combating / treating aging? In the last two decades, research on the molecular mechanisms of aging has led to a paradigm shift to such an extent that we can now say that aging is not an unalterable process ${ }^{13}$. The alternative approach to the prevention of disease in older people, promoted by Olshansky and colleagues ${ }^{11,12}$, has been termed the "dividend of longevity." By delaying the aging process, it should be possible to delay all agerelated and disability-related illnesses, such as AD rather than attacking them one byone, which is the approach of the current disease-based process of drug development ${ }^{14}$. Therefore, any benefits from the treatment and diagnosis of a single illness on life expectancy in older people are marginal $^{14,15}$. In this way, aging research is the ultimate form of preventative medicine: if the rate of aging can be slowed, then many of the diseases and declines in function associated with aging should be simultaneously delayed ${ }^{9}$. Fortunately, understanding the molecular mechanisms of aging has progressed rapidly in recent decades. A number of pathways affecting metabolism, growth, inflammation, and epigenetic modifications that alter the rate of aging and incidence of age-related diseases have been identified ${ }^{13,14,16}$ - Interventions that target the molecular mechanisms of aging have the potential to increase both life span and health span, which can be defined as the period of life free from chronic disease and disability ${ }^{9,17}$. In fact, accumulating scientific evidence from studies conducted in various organisms and species suggests that targeting aging will not just postpone chronic diseases but also prevent multiple age-associated metabolic alterations while extending healthy lifespan ${ }^{9,17,18,19,20}$. Among the most promising strategies that could be tested in humans for their effects on healthspan, are: (i) dietary interventions mimicking chronic dietary restriction (periodic fasting mimicking diets, protein restriction, etc.); (ii) pharmacological inhibition of the growth hormone/insulin growth factor-I axis; (iii) pharmacological inhibition of the mTOR-S6K pathway; (iv) drugs that activate AMPK or specific sirtuins; (v) use of spermidine and other epigenetic modulators; (vi) pharmacological inhibition of inflammation. These choices were based in part on consistent evidence for the pro-longevity effects and ability of these interventions to prevent or delay multiple age-related diseases and improve healthspan in simple model organisms and rodents and their potential to be safe and effective in extending human healthspan $18,21,22,23,24$.
Acting in mechanisms/targets of aging to delay Alzheimer's disease

The development of new interventions and pharmacological targets, based on the biology of aging, is a priority for humans and an major opportunity for the pharmaceutical industry ${ }^{25}$. However, the question is whether this can be achieved in a timely manner, since the assessment of longevity in humans requires decades, and the development of pharmacotherapies for standard illnesses has been going on for more than a decade. The availability of short-lived animal models has allowed the implementation of interventions that reduce, as far as possible, aging at different levels, acting at different molecular targets, and slowing down or delaying diseases associated with aging. We have recently revised the interventions with antiaging properties able to alter the onset and / or progression of AD. In this context, we described the antiaging therapies best studied in animal models of $\mathrm{AD}$, and which of these therapies are being applied in clinical trials ${ }^{26}$.We can classify these therapies into four groups: pharmacological therapies, lifestylerelated, molecular (genetic and epigenetic) and cellular. Different anti-aging interventions have been shown to prevent (and in some cases possibly restore) several parameters recognised as central symptoms to the development of $\mathrm{AD}^{26}$. In addition, they are taking the first steps towards translating these laboratory discoveries into clinical applications ${ }^{26}$. Some examples are shown:

\section{mTOR inhibitors}

Overwhelming data suggest that decreasing the activity of the mammalian target of rapamycin (mTOR) increases lifespan and healthspan. mTOR is a serine/threonine protein kinase, which is involved in the regulation of both protein synthesis and degradation, longevity and cytoskeletal formation ${ }^{27,28}$.The mTOR pathway is involved in the regulation of a wide repertoire of cellular functions in the brain and its dysregulation is emerging as a leitmotif in a large number of neurological disorders. In $A D$, altered mTOR signaling contributes to the inhibition of autophagy, deposition of $A \beta$ and tau aggregates and to the alteration of several neuronal metabolic pathways ${ }^{29}$. mTOR hyperactivity is observed in AD brains from human and mouse models and sufficient proof has demonstrated that alterations of mTOR may be one of the leading events contributing to the formation of toxic aggregates during $\mathrm{AD}$ pathology ${ }^{30,31}$. Moreover, current findings support the role of mTOR inhibitors (rapamycin, rapalogues) as potential therapeutic agents able to reduce $\mathrm{AD}$ hallmarks and recover cognitive performances ${ }^{29}$.Therefore, modulating mTOR activity is an attractive avenue to discover new therapies to enhance healthy brain and body aging, as well as attenuating age-related disease progression and treating age-related diseases, including AD. 


\section{Metformin}

Metformin is a biguanide that is used as a treatment for type 2 diabetes because it decreases hepatic gluconeogenesis and increases insulin sensitivity ${ }^{32}$. In addition, it is a powerful indirect activator of the AMP-activated protein kinase (AMPK). This enzyme is a critical regulator of energy homeostasis, and inhibits mTOR. It also activates the transcription factors SKN-1 / Nrf2, whose targets are diverse antioxidant genes ${ }^{32}$. In addition, metformin increases both lifespan and healthspan in different strains of mice ${ }^{33}$.The exact mechanism of metformin's advantageous activity in $\mathrm{AD}$ is not fully understood, but metformin is a drug that has an evidence based impact on neuroprotection via the activation of the AMPK pathway in human neural stem cells (hNCS's) ${ }^{34}$. Activation of AMPK-dependent pathways in hNCS's is a potential therapeutic target for AD, because cellular mechanisms of energy homeostasis are connected to AD pathogenesis ${ }^{34}$. Metformin was also found to markedly decrease Beta secretase 1 (BACE1) protein expression and activity in cell culture models and in vivo ${ }^{35}$, thereby reducing BACE1 cleavage products and the production of $A \beta$. In regard to the beneficial effects of metformin, its anti-inflammatory and antioxidative properties cannot be omitted. However, additional studies are needed to gain insight into the involvement of metformin in the regulation of tau associated with AD.

\section{Resveratrol}

Resveratrol, a polyphenol found in red grapes and certain berries, has received considerable attention for its reported ability to extend life span in some model organisms and be protective in animal models of age-related disease including neurodegeneration in rodent models of AD. These effects of resveratrol may be mediated through activation of the NAD-dependent protein deacetylase Sirtuin 1, which affects transcriptional regulation ${ }^{36}$. Moreover, recently Moussa and colleagues provided evidence that resveratrol impacts neuroinflammation when given to patients with mild or moderate $\mathrm{AD}^{37}$.

\section{Lifestyles}

Moderate physical exercise also produces numerous brain-beneficial effects in murine AD models. Among them we highlight, delayed neurodegeneration in the hippocampus, reduction of proinflammatory factors, increase of BDNF, vascular endothelial growth factor (VEGF) and IGF-1, as well as reduction of $A \beta^{38}$ and decreased neuroinflammation, which is why it improves the spatial memory in these mice ${ }^{39}$. Likewise, numerous studies have focused on the health benefits of the Mediterranean diet and its various components, specifically extra virgin olive oil (EVOO). Among the minor constituents of EVOO, a number of polyphenols and secoiridoids, including oleocanthal, hydroxytyrosol and oleuropein aglycone, are able to modulate many pathways that are relevant to the aging process. The beneficial effects of some components of EVOO in preventing and delaying the onset of $\mathrm{AD}$, and in reducing the severity of its symptoms, have been found in transgenic mouse models ${ }^{40,41}$. On the other hand, the population cohort study, Nurses' Health Study, showed that greater adherence to the Mediterranean diet was associated with longer telomeres ${ }^{42}$.

\section{TERT}

In aging, many genes have been described whose products are deregulated. One of the most studied is reverse transcriptase of telomerase (TERT). In a study in which TERT was overexpressed, it was possible to increase longevity in mice without producing adverse effects ${ }^{17}$. With this gene therapy the wear of telomeres (one of the markers of aging) is avoided and, therefore, prevent their shortening. TERT is present in the brain during its development, but not in adult neurons, and also has neuroprotective properties in animal models with neurodegenerative disorders, such as $\mathrm{AD}$. The length of telomeres is significantly lower in mice and in patients with $\mathrm{AD}$, compared to controls ${ }^{43,44}$. This indicates that gene therapies that raise TERT in the brain may protect against age-related neurodegeneration, and thus prevent $\mathrm{AD}^{45}$.

\section{Conclusions}

Most anti-aging interventions that increase life expectancy and / or duration of health in animal models have a therapeutic action in AD. There is currently no ideal anti-aging intervention that can correct, slow or reverse the course and / or pathology of AD. According this, therapy must address multiple targets within the network underlying the pathophysiology of $\mathrm{AD}$, with the idea that a combination therapy can create an effect that may be additive, multiplicative or synergistic. Therefore, several therapeutic interventions (by optimizing the balance between their beneficial and undesirable effects) and new research approaches in aging will be necessary to gain control of this devastating disease.

\section{Funding}

The authors received no financial support for the research, authorship, and/or publication of this article.

\section{Declaration of competing interests}

The authors report no competing interests with respect to the research, authorship, and/or publication of this article.

\section{References}

1. Bredesen DE. Reversal of cognitive decline: a novel therapeutic program. Aging (Albany NY). 2014; 6(9): 707-717.

2. Wisniewski T, Goñi F. Immunotherapeutic approaches for Alzheimer's disease. Neuron. 2015; 85(6): 1162-1176. 
3. Sperling R, Salloway S, Brooks DJ, et al. Amyloid-related imaging abnormalities in patients with Alzheimer's disease treated with bapineuzumab: a retrospective analysis. Lancet Neurol. 2012; 11(3): 241-249.

4. Wilcock DM, Colton CA. Immunotherapy, vascular pathology, and microhemorrhages in transgenic mice. CNS Neurol Disord Drug Targets. 2009; 8(1): 50-64.

5. Boche D, Nicoll JA. The role of the immune system in clearance of Abeta from the brain. Brain Pathol. 2008; 18(2): 267-278.

6. Toepper M. Dissociating normal aging from Alzheimer's disease: A view from cognitive neuroscience. J Alzheimers Dis. 2017; 57(2): 331-352.

7. Martin GM. Geroscience: Addressing the mismatch between its exciting research opportunities, its economic imperative and its current funding crisis. Exp Gerontol. 2017; 94: 46-51.

8. Hung WW, Ross JS, Boockvar KS, et al. Recent trends in chronic disease, impairment and disability among older adults in the United States. BMC Geriatr. 2011; 11: 47.

9. Kaeberlein M. The biology of aging: Citizen scientists and their pets as a bridge between research on model organisms and human subjects. Vet Pathol. 2016; 53(2): 291-298.

10. Kaeberlein M. Longevity and aging. F1000Prime Rep. 2013; 5:5.

11. Olshansky SJ, Perry D, Miller RA, et al. In pursuit of the longevity dividend: What should we be doing to prepare for the unprecendented aging of humanity. The Scientist. 2006; 20: 28-36.

12. Olshansky SJ, Perry D, Miller RA, et al. Pursuing the longevity dividend: scientific goals for an aging world. Ann N Y Acad Sci. 2007; 1114: 11-13.

13. Lopez-Otin C, Blasco MA, Partridge L, et al. The hallmarks of aging Cell. 2013; 153(6): 1194-1217.

14. Sierra F. The Emergence of Geroscience as an Interdisciplinary Approach to the Enhancement of Health Span and Life Span. Cold Spring Harb Perspect Med. 2016; 6(4): a025163.

15. Welch HG, Albertsen PC, Nease RF, et al. Estimating treatment benefits for the elderly: the effect of competing risks. Ann Intern Med. 1996; 124(6): 577-584.

16. Figueira I, Fernandes A, Mladenovic Djordjevic A, et al. Interventions for age-related diseases: Shifting the paradigm. Mech Ageing Dev. 2016;160: 69-92.

17. Bernardes de Jesus B, Vera E, Schneeberger K, et al. Telomerase gene therapy in adult and old mice delays aging and increases longevity without increasing cancer. EMBO Mol Med. 2012; 4(8): 691-704.

18. Longo VD, Antebi A, Bartke A, et al. Interventions to slow aging in humans: Are we ready. Aging Cell. 2015;14(4): 497-510.

19. Melov S. Geroscience approaches to increase healthspan and slow aging. F1000Res. 2016; 29: 5.

20. Seals DR, Justice JN, LaRocca TJ. Physiological geroscience: targeting function to increase healthspan and achieve optimal longevity. J Physiol. 2016; 594(8): 2001-2024.

21. Fontana L, Partridge L, Longo VD. Extending healthy life span-from yeast to humans. Science. 2010; 328(5976): 321-326.

22. Armanios M, de Cabo R, Mannick J, et al. Translational strategies in aging and age-related disease. Nat Med. 2015; 21(12): 1395-1399.

23. Newman JC, Milman S, Hashmi SK, et al. Strategies and challenges in clinical trials targeting human aging. J Gerontol A Biol Sci Med Sci. 2016; 71(11): 1424-1434

24. Espeland MA, Crimmins EM, Grossardt BR, et al. Clinical trials targeting aging and age-related multimorbidity. J Gerontol A Biol Sci Med Sci. 2017; 72(3): 355-361.

25. Le Couteur DG, Sinclair DA. A blueprint for developing therapeutic approaches that increase healthspan and delay death. J Gerontol A Biol Sci Med Sci. 2010; 65(7): 693-694.

26. Alonso Abreu GS, Brito-Armas JM, Castro-Fuentes R. Anti-ageing therapies in Alzheimer's disease. Rev Esp Geriatr Gerontol. 2017; doi: 10.1016/j.regg.2017.02.007.

27. Wullschleger S, Loewith $\mathrm{R}$, Hall MN. TOR signaling in growth and metabolism. Cell. 2006; 124: 471-484.

28. Johnson SC, Rabinovitch PS, Kaeberlein M. mTOR is a key modulator of ageing and age-related disease. Nature. 2013; 493: 338-345.

29. Tramutola A, Lanzillotta C, Di Domenico F. Targeting mTOR to reduce Alzheimer-related cognitive decline: from current hits to future therapies. Expert Rev Neurother. 2017; 17(1): 33-45.

30. Oddo $\mathrm{S}$. The role of mTOR signaling in Alzheimer disease. Front Biosci (Schol Ed). 2012; 4: 941-952.

31. Maiese K. Taking aim at Alzheimer's disease through the mammalian target of rapamycin. Ann Med. 2014; 46(8): 587-596.

32. Berstein LM. Metformin in obesity, cancer and aging: addressing controversies. Aging (Albany NY). 2012; 4(5): 320-329.

33. Martin-Montalvo A, Mercken EM, Mitchell SJ, et al. Metformin improves healthspan and lifespan in mice. Nat Commun. 2013; 4: 2192. doi: 10.1038/ncomms3192.

34. Chiang MC, Cheng YC, Chen SJ, et al. Metformin activation of AMPKdependent pathways is neuroprotective in human neural stem cell against amyloid-beta-induced mitochodrial dysfunction. Exp Cell Res. 2016; 347: 322-31.

35. Markowicz-Piasecka M, Sikora J, Szydłowska A, et al. Metformin - a Future Therapy for Neurodegenerative Diseases. Pharm Res. 2017. doi: 10.1007/s11095-017-2199-y.

36. Martin I. Resveratrol for Alzheimer's disease. Sci Transl Med. 2017; $9(375)$. doi: 10.1126/scitranslmed.aam6055.

37. Moussa C, Hebron M, Huang X, et al. Resveratrol regulates neuroinflammation and induces adaptive immunity in Alzheimer's disease. J Neuroinflammation. 2017; 14(1):1. doi: 10.1186/s12974016-0779-0.

38. Hüttenrauch M, Brauß A, Kurdakova A, et al. Physical activity delays hippocampal neurodegeneration and rescues memory deficits in an Alzheimer disease mouse model. Transl Psychiatry. 2016; 6: e800. doi: 10.1038/tp.2016.65.

39. Spielman LJ, Little JP, Klegeris A. Physical activity and exercise attenuate neuroinflammation in neurological diseases. Brain Res Bull. 2016; 125: 19-29.

40. Grossi C, Rigacci S, Ambrosini S, et al. The polyphenol oleuropein aglycone protects TgCRND8 mice against Aß plaque pathology. PLoS One. 2013; 8(8): e71702.

41. Peng Y, Hou C, Yang Z, et al. Hydroxytyrosol mildly improve cognitive function independent of APP processing in APP/PS1 mice. Mol Nutr Food Res. 2016; 60(11): 2331-2342.

42. Crous-Bou M, Fung TT, Prescott J, et al. Mediterranean diet and telomere length in Nurses' Health Study: population based cohort study. BMJ. 2014; 349: g6674. doi: 10.1136/bmj.g6674.

43. Franco S, Blasco MA, Siedlak SL, et al. Telomeres and telomerase in Alzheimer's disease: epiphenomena or a new focus for therapeutic strategy. Alzheimers Dement. 2006; 2(3):164-168.

44. Forero DA, González-Giraldo Y, López-Quintero C, et al. Meta-analysis of Telomere Length in Alzheimer's Disease. J Gerontol A Biol Sci Med Sci. 2016; 71(8):1069-1073.

45. Rolyan H, Scheffold A, Heinrich A, et al. Telomere shortening reduces Alzheimer's disease amyloid pathology in mice. Brain. 2011; 134(Pt 7): 2044-2056 